\title{
Dynamic Dual Polarized GNSS Reflectometry Using UAV
}

\author{
Ankit Regmi ${ }^{1}$, Tuomo Hänninen ${ }^{1}$, Marko E. Leinonen ${ }^{1}$, Aarno Pärssinen ${ }^{1}$, Markus Berg ${ }^{1}$ \\ Centre for Wireless Communications ${ }^{1}$, University of Oulu, Oulu, Finland, ankit.regmi@oulu.fi
}

\begin{abstract}
In this paper, GNSS-Reflectometry is performed using Unmanned Aerial Vehicle (UAV). Received GNSS multipath signal is utilized to perform environment remote sensing in dynamic and static flight modes. Right-Hand Circular Polarized (RHCP) multipath signal is recorded and its interference frequency is used to estimate the height of UAV above the ground surface. In the dynamic flight mode, the $\mathrm{C} / \mathrm{N} 0$ level of the reflected Left-Hand Circular Polarized (LHCP) signal is used to indicate the presence of different ground surfaces. In comparison with the ground measurement systems, UAV provides a dynamic and flexible measurement platform, potentially significantly reducing the measurement time. At the same time, measurement signal quality is influenced due to the variation of the UAV altitude. The results presented strongly indicate the possibility of utilizing UAVs as measurement platform for remote sensing using GNSS multipath signal.

Index Terms-GNSS-R, multipath, UAV, Drones, dual circular
\end{abstract} polarization, reflection, remote sensing.

\section{INTRODUCTION}

Global Navigation Satellite System (GNSS) signals are freely available around the globe and can be captured using compatible GNSS receivers. Four major GNSS constellations that have at least 20 active satellites, providing coverage worldwide are GPS (USA), GLONASS (Russia), BeiDou (China), Galileo (Europe). At least four satellites with line-ofsight (LOS) propagation is required to get a good positioning solution. However, when the GNSS signals arrive at the earth surface, the influence of environment on these signals could be significant. Urban environment or rapidly changing landscape (forests or mountain valleys) can cause the GNSS signals to undergo fundamental wave propagation effects such as reflection, diffraction, and scattering. These signals arrive at the receiver via different paths to create a multipath signal. Multipath signals significantly degrade receiver performance and introduce large errors in positioning solution. Therefore, the multipath signal is an unwanted quantity in the GNSS navigation and positioning systems. However, the analysis of GPS reflected signal from the sea in [1], gave the altitude information of the aircraft. Henceforth, the possibility to utilize the GNSS multipath signal for environment sensing was realized. Few years later, [2] established the theoretical foundation for GNSS-Reflectometry (GNSS-R) and ever since several theories and applications have been developed in the field of GNSS-R remote sensing.

GNSS-R is a passive remote sensing technique used to estimate various environment parameters by analysing GNSS multipath signals. GNSS-R has been used to estimate sea level, sea-wave height, wind speed over the ocean, snow and ice thickness, soil moisture etc [3]-[8]. The incident right hand circular polarized (RHCP) signal becomes left hand circular polarized (LHCP) after reflection from smooth surface, for satellite elevation angles greater than the Brewster angle. For angles less than the Brewster angle, the reflected signal will still be RHCP. The reflected RHCP and direct signals add up creating a pattern of constructive and destructive interference. This interference pattern is created due to the angular motion of satellite causing continuous change in the excess path length travelled by the reflected signal with respect to incident signal. This change in polarization is utilized to extract environment properties. Therefore, the Dual Circular Polarized Reception (DCPR) system is used to receive RHCP and LHCP signals, simultaneously [4]. GPS L1 C/A and GLONASS L1OF transmitted RHCP signals are used in this study. The DCPR system is installed on board an Unmanned Aerial Vehicle (UAV), that provides unprecedented flexibility to the measurement setup. Dynamic GNSS-R on board an airplane has been performed to identify water bodies in [9]. However, this paper investigates the possibility to use UAVs as a measurement platform to perform GNSS-R faster, with low complexity, and as a more environment friendly approach. Thus, we proposes an adaptive and mobile GNSS-R measurement station. GNSS$\mathrm{R}$ measurements are performed at sea and lake for estimating the height and sensing different surfaces, respectively. GNSS$\mathrm{R}$ measurements are performed in static as well as dynamic modes.

This paper is arranged in the following way. A brief introduction on GNSS-R is given in Section II. Section III gives a detailed description of DCPR integrated drone measurement system and setup. The measurement results and analysis are presented in Section IV. Sources of uncertainty is discussed in section $\mathrm{V}$ and the paper is concluded in Section VI.

\section{THEORETICAL BACKGROUND OF GNSS-REFLECTOMETRY}

The direct and reflected GNSS RHCP signals add up to create an interference pattern which is a function of height of the antenna above the reflecting surface and satellite elevation angle. The polarization of the direct and reflected signal has to be same to create this interference pattern. Therefore, it is only possible to capture these interference patterns for lower elevation angles that are closer to the Brewster angle. The interference pattern is due to the satellite movement in the elevation plane that continuously changes the phase difference 
between direct and received signal. This phase difference is due to the excess path length travelled by the reflected signal with respect to the direct signal. The excess path length travelled by the reflected signal is given by [10],

$$
\delta_{\text {epl }}=2 h \sin \theta
$$

Here, $h$ is the height of the antenna phase centre from the horizontal reflecting surface and $\theta$ is the satellite elevation angle. The phase change due excess path length is given by,

$$
\phi_{e p l}=\frac{2 \pi \delta_{e p l}}{\lambda}
$$

Here, $\lambda$ is the carrier wavelength i.e. approx $19 \mathrm{~cm}$. Differentiating Eq. 2 with respect to $\sin \theta$ and dividing the right-hand side by $\frac{1}{2 \pi}$ gives us the constant interference frequency term. Thus, the interferometric reflectometry frequency is given by [11],

$$
f_{I R}=\frac{1}{2 \pi} \frac{\partial \phi_{e p l}}{\partial \sin \theta}=\frac{2 h}{\lambda}
$$

Therefore, from Eq. 3, the interference frequency is directly proportional to the height of the antenna and is a function of rate of change of sine of satellite elevation angle. In practice, the GNSS satellites move in a relatively slow and constant angular motion. They appear to be moving slower near the horizon and faster near the zenith. Therefore, the number of interference fringes is mainly dominated by the height of the antenna, which is the key parameter to perform GNSSInterferometric Reflectometry (GNSS-IR).

The reflected signal is the function of carrier frequency, satellite elevation angle, and relative permittivity of the reflecting surface. When specular reflection occurs, the signal amplitude and phase changes with respect to the Fresnel reflection coefficient. The reflected signal for elevation angles greater than Brewster angle will be LHCP. A significant change in reflected LHCP signal strength indicates change in the electromagnetic properties of the reflecting surface. Therefore, characteristics of reflected signal can be used to identify surface by estimating the surface electromagnetic properties.

\section{DRONE ASSISTED DCPR SYSTEM AND MEASUREMENT SETUP}

GNSS-R was performed using DCPR system on board high performance UAV. The UAV used was DJI Matrice 210 V2 RTK. The DCPR system consisted of a dual circular polarized antenna connected to two GNSS receivers to simultaneously receive RHCP and LHCP signals [4]. The dual circular polarized antenna with cross-polarization discrimination (XPD) greater than $25 \mathrm{~dB}$ for boresight and port isolation greater than $15 \mathrm{~dB}$ was used [12]. The low cost, light weight u-blox NEOM8T USB GNSS receivers were connected to and powered by the on board drone computer. The GNSS evaluation software and the on board computer were remotely controlled via LTE network using a computer on the ground. The measurement setup is shown in Fig. 1. The DCPR system weighs less than a kilogram, and therefore is suitable to be used on board UAVs.

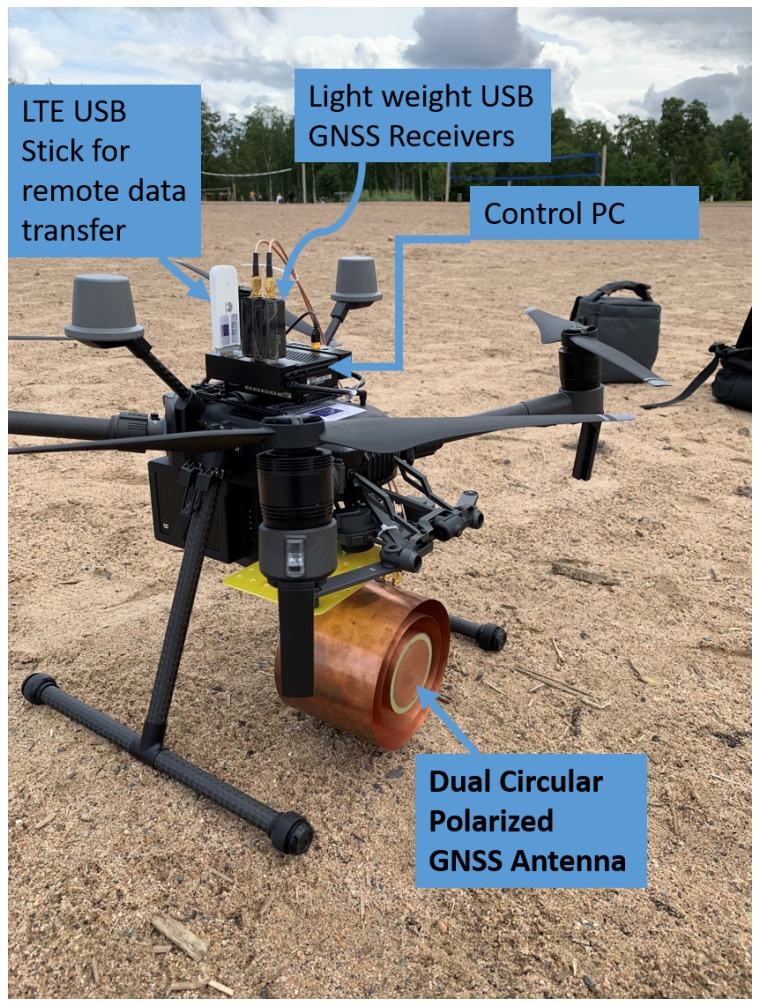

Fig. 1: GNSS-R Drone Measurement System

The receivers recorded carrier-to-noise density ratio (C/N0) data, satellite elevation and azimuth angle at rate of 1 sample/second. The measurement resolution was $1 \mathrm{~dB}-\mathrm{Hz}$ for $\mathrm{C} / \mathrm{N} 0$ data, and $1^{\circ}$ for elevation and azimuth angles, respectively. Altitude data from the drone was used as the ground truth data for comparison. Drone's flight data provided other parameters used for dynamic measurement such as heading, flight path, velocity, distance travelled from take-off point.

Static as well as dynamic measurements were performed at two different locations in Oulu, Finland. Static measurements were performed at Bay of Bothnia in Baltic Sea and the location for dynamic measurement was at Pyykösjärvi lake. The coordinates for measurement site were $65^{\circ} 01^{\prime} 55.6916^{\prime \prime} \mathrm{N}$, $25^{\circ} 24^{\prime} 28.4178^{\prime \prime} \mathrm{E}$ and $65^{\circ} 02^{\prime} 57.2123^{\prime \prime} \mathrm{N}, 25^{\circ} 29^{\prime} 32.1667^{\prime \prime} \mathrm{E}$, respectively.

The use of UAV gave the advantage to manipulate drone altitude, velocity and heading significantly faster as required by the measurement. Thus, it is an adaptive and mobile GNSS$\mathrm{R}$ measurement station.

\section{ANALYSis OF RECORDED DATA}

Static and dynamic UAV data recordings were performed in different environments including land, lake, and sea areas. Performance of static measurement is compared with a seaside located stationary measurement system.

\section{A. Static Measurement}

Static measurements was performed at sea. The main objective of the static setup was to perform GNSS-IR experiment 

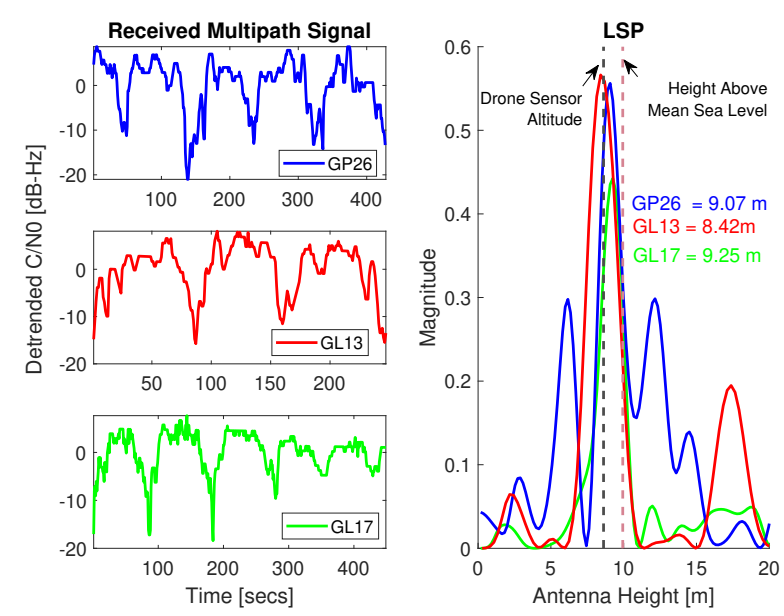

(a) Static Flight 1
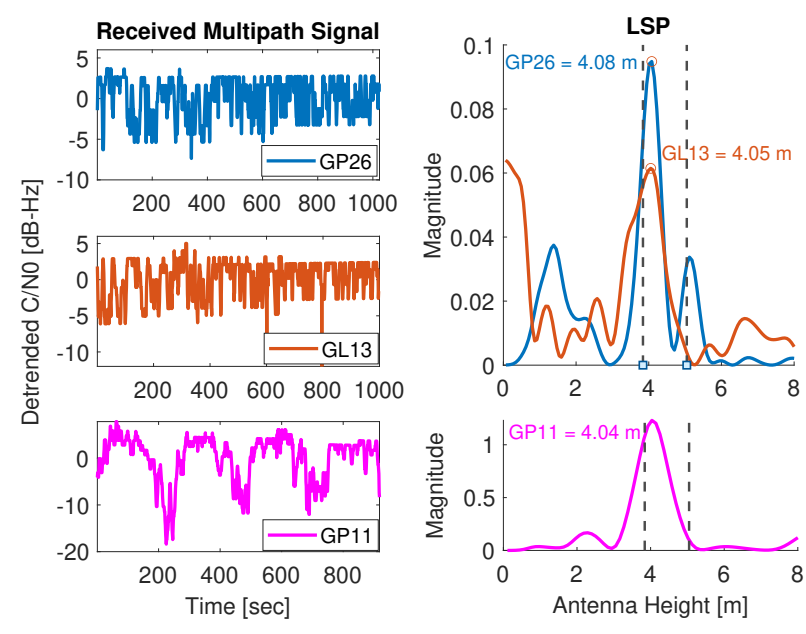

(b) Static flight 2

Fig. 2: GNSS Altimetry for Sea Surface Measurements

to derive the height of the UAV above the reflecting surface. Previous studies mainly used tripods or fixed ground stations to perform GNSS-IR, where the time series measurements lasted few hours to even months. These long time series campaigns give information about the surface properties for longer duration of periods. For example, accumulation of snow over the winter, sea-level height for certain period of time, soil moisture content during dry and wet seasons, etc. However, our focus here is to achieve similar goals with significantly reduced measurement time. The use of drones to perform GNSS-IR helps us in achieving our goal.

The GNSS-IR drone measurement system (DMS) was flown over water. Drone's heading was selected based on best satellites elevation and azimuth relative to the drone. The drone hovered at a suitable altitude and the recording of GNSS data was started. The RHCP GNSS-IR received signal over sea and the derived antenna height for satellites GP26, GL13 and GL17 is shown in Fig. 2a. Similarly, for the second flight a different altitude was chosen. The GNSS-IR results for satellites GP26, GL13 and GP11 are shown in Fig. 2b. Here, GP stands for GPS satellites and GL for GLONASS. The effect of LOS signal is removed from the original C/NO data using the antenna radiation pattern and is presented as detrended C/N0 in Fig. 2 [7], [13].

The right-hand side in Fig. 2a and 2b is the Lomb-Scargle Periodogram (LSP) of the multipath signal represented as the function of antenna height above the reflecting surface. LSP performs Fast Fourier Transform (FFT) of the received multipath signal with respect to sine of satellite elevation angle [13]. The frequency of the multipath signal is related to the antenna height using Eq. 3. In Fig. 2, the two constant vertical lines denote the mean altitudes given by the drone flight data. The lower vertical line is the drone's sensor derived height and upper bound is GPS derived height above mean sea level. The GNSS-IR altimetry agrees well with the ground-truth data.

In Fig. 2a, the satellite elevation angle was less than $5^{\circ}$ for all satellites. It is observed that more than 3 interference notches were enough to extract the antenna height with acceptable accuracy in less than 9 minutes. The LSP of GL13 underestimated the height due to less number of interference fringes. Therefore, more the number of interference fringes better the estimate of the frequency content of the multipath signal. On the contrary, to achieve same number of interference fringes, more than twice the time was required as shown in Fig. $2 \mathrm{~b}$ for GP11. Here, the altitude was approx. half as compared to the first flight. However, more number of samples gives better resolution, hence better accuracy and can be seen in Fig. 2b. A clear interference pattern was not visible in the received multipath signal for angles greater than $6^{\circ}$ as can be seen in case of GP26 and GL13 in Fig. 2b. This could be due to the placement of the antenna. The direct signal at higher elevation angles could have been affected by drone's upper body and rotors. However, the LSP for GP26 and GL13 was able to capture the dominant peak with accurate antenna height. Comparing the LSPs for the second flight, the magnitude of LSP in case of GP11 is 10 times higher than for GP26 and GL13, where the interference patterns were not clearly visible.

\section{B. Dynamic Measurement}

Dynamic measurement was performed at Pyykösjärvi lake. The measurement surrounding was significantly different compared to the open sea scenario. The measurement site consisted of forest, sand beach, vegetation on the shore, and a lake. The drone was flown back and forth four times over sand beach, vegetation and lake at approx. altitude of $4.5 \mathrm{~m}$. The specular point of reflection with respect to the strength of received LHCP reflected signal for dynamic flight is shown in Fig. 3. The specular point has been plotted taking into account the satellite elevation as well as azimuth angle, according to [13]. Specular points for satellites with different azimuth angles are chosen for clarity. 


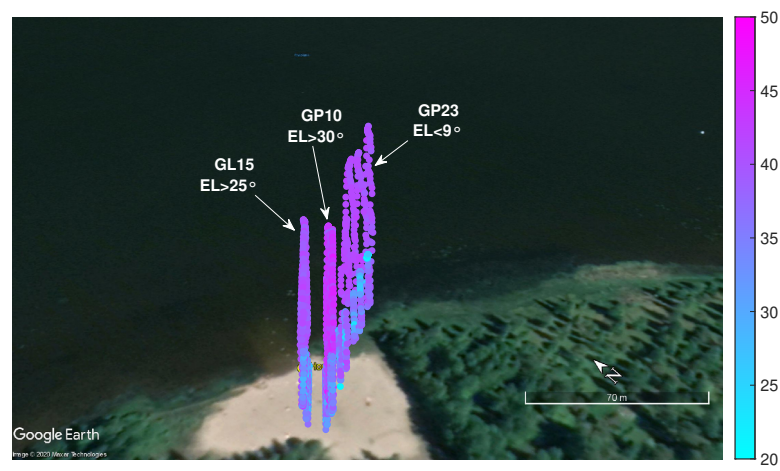

Fig. 3: Specular Points for Dynamic Flight

In Fig. 3, it is observed for GP23 that the specular point is sensitive to azimuth movement when the satellite is at low elevation, whereas for higher elevation satellite GP10 and GL15, the specular point is close to the antenna and is less sensitive to azimuth variation. The reflected signal strength (reflection coefficient) is the function of elevation angle and complex permittivity of reflecting medium. The real part of permittivity is given in Table I. It can be observed that reflection is strong when the drone is over water. But as drone approaches the vegetation, the signal attenuation is significant. Vegetation area significantly reduces the intensity of reflected power in specular direction, even if they have high permittivity values. For specular reflection to occur, the reflecting surface area should be relatively smooth, flat and larger than the First Fresnel zone (FFZ) [14]. The vegetation area fails to fulfil the criteria for specular reflection to occur. The specular reflection dominates again as the drone flies over the flat sand beach resulting in increase in reflected signal strength.

The reflected LHCP signal along with the RHCP C/NO data for dynamic measurement is shown in Fig. 4 for satellites GP10, GP23, GL16, respectively. The RHCP signal for GP10 is stable and linearly increasing whereas RHCP C/NO for GP23 and GL16 are of multipath nature. GP23 and GL16 are at low elevation angles ranging from $6^{\circ}$ to $9^{\circ}$ and $10^{\circ}$ to $13^{\circ}$, respective and the multipath is due to interference from the reflected RHCP signal. As described in Section II and IV A, the reflected signal is still RHCP near Brewster angle and becomes LHCP at angles greater than Brewster angle. The elevation range for GP10 was ranges from $33^{\circ}$ to $37^{\circ}$, therefore, signal is mostly direct RHCP signal with negligible reflected component.

Repeated pattern in received signal strength is witnessed for reflected LHCP signal for dynamic flight. The variation of reflected signal level with respect to the reflecting surface can

TABLE I: Relative Permittivities at L1 band [15]

\begin{tabular}{|c|c|c|}
\hline Water (Lake) & Vegetation & Sand \\
\hline 80 & $7-38$ & $7-15$ \\
\hline
\end{tabular}
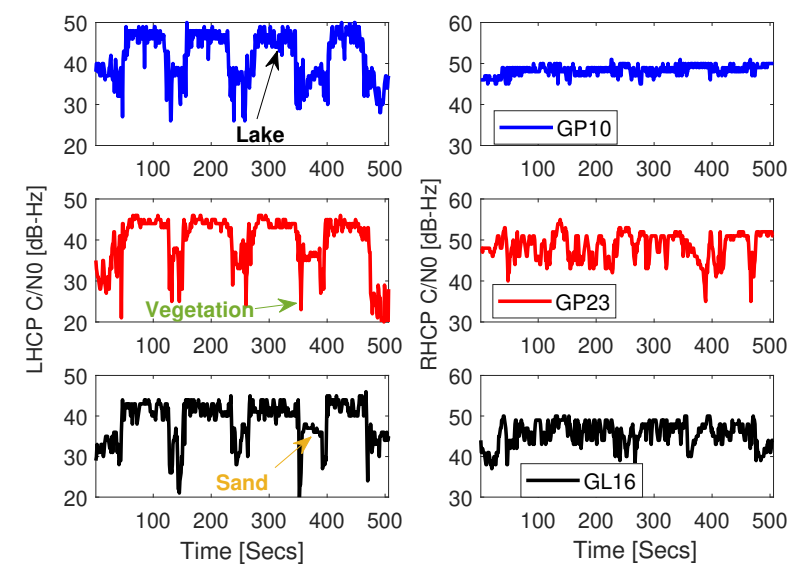

Fig. 4: Received LHCP and RHCP C/NO data

be mapped accurately in Fig. 3. The characteristics of reflected signal from their respective specular footprints gave a clear indication of nature of reflecting surface. The identification of different surfaces based on reflected LHCP signal level is shown by arrows in Fig. 4. Thus, the analysis of flight path and signal level in Fig. 3 and Fig. 4 gives a very strong indication of nature of reflecting surfaces and is a key application in remote sensing.

\section{Sources of Data UnCERTAinty}

The DMS system provides significant flexibility compared to ground measurement systems. However, this flexibility comes with some cost. First source of uncertainty arises from the fact that, even the drone is relatively stable, it is not completely static. In fact, the drone is hovering and always trying to maintain the input altitude. The drone altitude data for static flight mode is presented in Fig. 5. There are two altitude parameters presented here, height above mean sea level, that is derived by drone's GPS system and height derived from drone's altitude sensor. It can be seen that the GPS derived height is stable as compared to the sensor height, whereas,

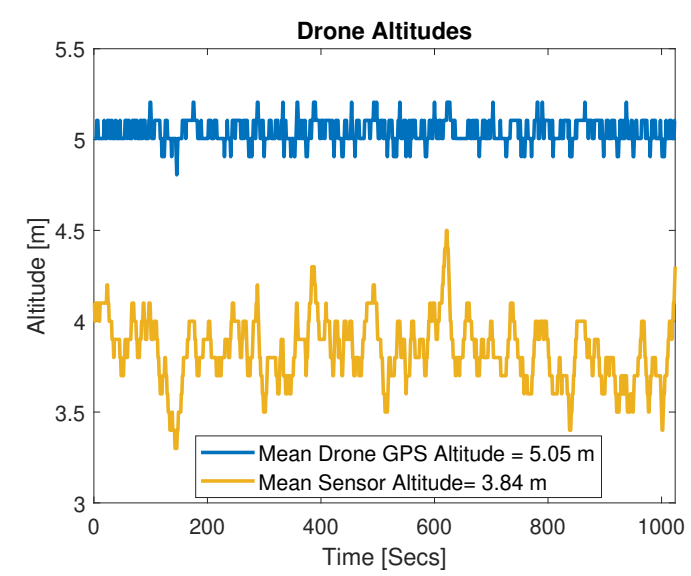

Fig. 5: Drone Altitude Variation 

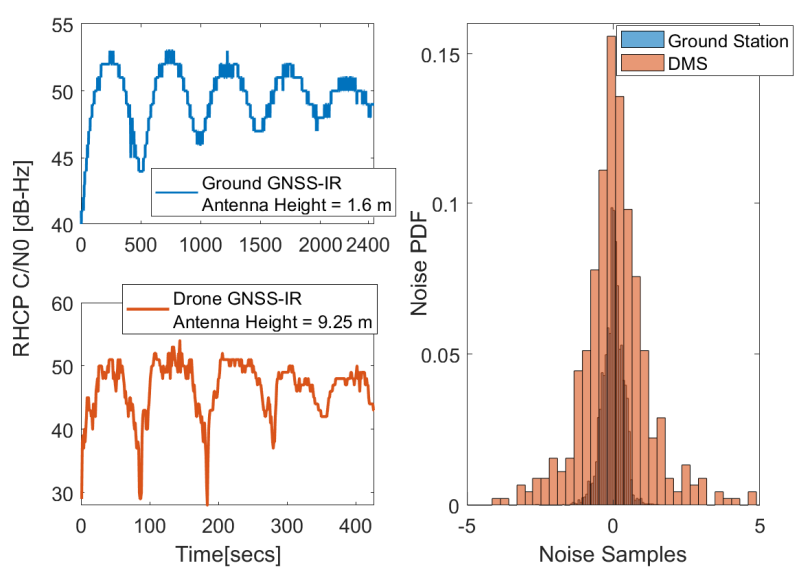

Fig. 6: Signal Quality Comparison

sensor height fluctuation is comparatively more and is due the sea surface variation. In any case, inaccuracies will result in the derived altimetry as the phase of the interference pattern is continuously altered by these small changes.

The quality of GNSS-IR C/N0 between fixed ground measurement system and DMS is compared and shown in Fig. 6. Ground station data is relatively stable, and clear constant interference fringes are visible, whereas in case of DMS the noise is increased. Due to the increased altitude of the drone, the increased interference frequency is observed again. The right-hand side of the figure is the histogram of the noise of the signal. The zero mean noise is carefully extracted using a highpass filter. It is clear that the background noise in the DMS is significantly higher than the fixed ground station case. In the GNSS-R DMS, apart from receiver internal noise, external noise from drone's rotors and vibration also contributes to the overall noise.

\section{CONCLUSION}

An adaptive and mobile measurement setup to perform GNSS-R using DCPR system on board UAV is proposed. This study shows that high performance drones can give unprecedented flexibility to perform GNSS-R measurements in significantly less time than previous ground based systems. However, the drone altitude variations introduce increased noise in GNSS-IR signal in comparison with the fixed ground stations. UAV integrated DCPR system was able to achieve GNSS-IR altimetry and GNSS-R surface sensing. GNSS-IR altimetry using RHCP multipath signal was performed in static setting, whereas surface sensing using LHCP reflected signal was done in dynamic mode. GNSS-IR altimetry at two different altitudes agreed well with the derived altitude from drone sensors and drone's GPS system. Reflected LHCP signal in dynamic flight mode was able to identify different surfaces that included water (lake), sand and vegetation area. Therefore, the proposed GNSS-R measurement platform using UAVs opens new possibilities for more clean, localized and instantaneous remote sensing applications.

\section{ACKNOWLEDGMENT}

This work was supported in part by the Academy of Finland 6Genesis Flagship (grant no. 318927) and 5G-VIIMA research project. InfoTech Oulu is also acknowledged for financial support through doctoral training position.

\section{REFERENCES}

[1] J. C. Auber, A. Bibaut, and J. M. Rigal, "Characterization of multipath on land and sea at GPS frequencies," in Proceedings of the 7th International Technical Meeting of the Satellite Division of The Institute of Navigation (ION GPS 1994), Salt Lake City, UT, September ,1994, pp. 1155-1171.

[2] M. Martin-Neira, "A passive reflectometry and interferometry system (paris): Application to ocean altimetry," ESA Journal, vol. 17, pp. 331$355,1993$.

[3] K. M. Larson, J. S. Löfgren, and R. Haas, "Coastal sea level measurements using a single geodetic gps receiver," Advances in Space Research, vol. 51, no. 8, pp. $1301-1310,2013$.

[4] A. Regmi, A. Pärssinen, and M. Berg, "Sea surface characterization using dual polarized GNSS reception system," in 2020 14th European Conference on Antennas and Propagation (EuCAP), 2020, pp. 1-5.

[5] M. B. Rivas and M. Martin-Neira, "Coherent GPS reflections from the sea surface," IEEE Geoscience and Remote Sensing Letters, vol. 3, no. 1, pp. 28-31, Jan 2006.

[6] K. M. Larson, "GPS interferometric reflectometry: applications to surface soil moisture, snow depth, and vegetation water content in the western united states," Wiley Interdisciplinary Reviews: Water, vol. 3, no. 6, pp. 775-787, 2016.

[7] A. Regmi, M. Berg, and A. Pärssinen, "A method for ice thickness characterization using GNSS C/N0 data," in Proceedings of 8th AsiaPacific Conference on Antennas and Propagation (APCAP), 2019, pp. $1-5$.

[8] A. Alonso Arroyo, A. Camps, A. Aguasca, G. F. Forte, A. Monerris, C. Rüdiger, J. P. Walker, H. Park, D. Pascual, and R. Onrubia, "Dualpolarization GNSS-R interference pattern technique for soil moisture mapping," IEEE Journal of Selected Topics in Applied Earth Observations and Remote Sensing, vol. 7, no. 5, pp. 1533-1544, May 2014.

[9] Y. Jia, P. Savi, D. Canone, and R. Notarpietro, "Estimation of surface characteristics using GNSS LH-reflected signals: Land versus water," IEEE Journal of Selected Topics in Applied Earth Observations and Remote Sensing, vol. 9, no. 10, pp. 4752-4758, Oct 2016.

[10] B. M. Hannah, "Modelling and simulation of GPS multipath propagation,” Ph.D. dissertation, Queensland University of Technology, 2001.

[11] Y. Georgiadou and A. Kleusberg, "On carrier signal multipath effects in relative gps positioning," Manuscripta geodaetica, vol. 13, no. 3, pp. 172-179, 1988.

[12] M. Berg, J. Chen, and A. Pärssinen, "Radiation characteristics of differentially-fed dual circularly polarized GNSS antenna," in 2019 13th European Conference on Antennas and Propagation (EuCAP), March 2019, pp. 1-5.

[13] K. M. Larson and F. G. Nievinski, "GPS snow sensing: results from the earthscope plate boundary observatory," GPS Solutions, vol. 17, no. 1, pp. 41-52, Jan 2013.

[14] C. Balanis, Advanced Engineering Electromagnetics. Wiley, 1989.

[15] ITU-R, "Electrical characteristics of the surface of the earth," International Telecommunications Union, vol. ITU-R Recommendation P.527-4, Jun 2017. 